\title{
Technologies to improve the solubility, dissolution and bioavailability of poorly soluble drugs
}

\begin{abstract}
Combinatorial chemistry, computational molecular modeling and high throughput screening in drug discovery have significantly increased the number of poorly soluble drugs. About $40 \%$ of drugs developed in the past and about $90 \%$ of the drugs in development are poorly soluble drugs. When administered orally, a drug has to first dissolve in gastrointestinal fluids before it can be absorbed in to the blood and reach its site of action. The objective of this review article is to outline the key aspects of the commonly used technologies to improve the solubility, dissolution, and bioavailability of poorly soluble drugs. The technologies covered in this article are particle size reduction (micronization and nanosuspensions), solid dispersions (spray drying and hot melt extrusion), lipid based delivery systems, and inclusion complexes. Jet mill and High pressure Homogenizer are used primarily for micronization and preparation of nano suspensions, respectively. Spray drier and Hot melt extruder are used for the preparation of solid dispersions. The majority of the lipid based formulations are filled into hard or soft gelatin capsules. Drug-cyclodextrin inclusion complexes are generally prepared by Coprecipitate and Spray drying methods. A number of poorly soluble drugs have been successfully introduced to the market by using new technologies. Among the available technologies, solid dispersion formulations and lipid based formulations have shown the most commercial success and continue to be widely employed. Physicochemical characteristics of the drug and advantages and disadvantages of different technologies need to be considered before selecting a technology. Advances being made in solubility enhancement technologies and sophisticated analytical techniques to measure the performance of the dosage forms in-vitro and in-vivo and new research in the area of modeling and simulation are expected to facilitate the development of new dosage forms that will successfully overcome the limitations of not only poorly soluble drugs but also poorly permeable drugs.
\end{abstract}

Keywords: poorly soluble drugs, micronization, nanosuspensions, spray drying, hot melt extrusion, lipid based delivery systems, inclusion complexes
Volume 7 Issue I - 2018

Narayan Kanikkannan

Millennial Pharma Solutions, USA

Correspondence: Narayan Kanikkannan, Millennial Pharma Solutions, LLC, 136 Windy Acres Estates Drive, Ballwin, MO 6302I, USA, Tel : +|-3|4-65I-3233;

Emailnkanikkannan@millennialpharma.com

Received: December 04, 2017 | Published: February 01, 2018
Abbreviations: SDD, spray dried dispersion; HPMCAS, hydroxypropyl methylcellulose acetate succinate; HPMC, hydroxypropyl methylcellulose; PVP, polyvinylpyrrolidone; PVA, polyvinylacetate; HME, hot melt extrusion; SEDDS, self-emulsifying drug delivery system; SMEDDS, self-microemulsifying drug delivery system; SNEDDS, self-nanoemulsifying drug delivery system; CD, cyclodextrin

\section{Introduction}

Oral administration of drugs is still the predominant route of administration due to its significant advantages including easy administration, high patient compliance, and cost effectiveness. When administered orally, a drug has to first dissolve in gastrointestinal fluids before it can be absorbed in to the blood and reach its site of action. Combinatorial chemistry, computational molecular modeling and high throughput screening in drug discovery have significantly increased the number of poorly soluble drugs. About $40 \%$ of drugs developed by Pharma industry and about $90 \%$ of the drugs in development pipeline are poorly soluble drugs. ${ }^{1}$ Drug solubility and bioavailability enhancement are the important challenges in the field of formulation of pharmaceuticals. Traditional pharmaceutical processes cannot be used for the development of dosage forms of poorly soluble drugs. New Technologies are needed to improve the solubility, dissolution, and bioavailability of poorly soluble drugs. This review provides an overview of the commonly used technologies to enhance the solubility, dissolution, and bioavailability of poorly soluble drugs, including their advantages and disadvantages.

\section{Technologies}

There are several technologies that have been successfully employed to enhance the solubility, dissolution, and bioavailability of poorly soluble drugs. ${ }^{2-8}$ This section discusses some of the commonly used technologies to improve the solubility, dissolution and bioavailability of poorly soluble drugs.

\section{a. Particle size reduction}

i. Micronization

ii. Nanosuspensions

\section{b. Solid dispersions}

Spray drying

Hot melt extrusion

\section{c. Lipid based delivery systems}

\section{d. Inclusion complexes}

\section{Particle size reduction}

The drug solubility is generally related to its particle size. The larger surface area allows greater interaction with the solvent, increasing the solubility. Size reduction involves breaking the drug particles into smaller ones by dry or wet milling. Particle size reduction can be achieved by micronization and nanosuspensions. Each technique utilizes different equipment for reduction of the particle size. 


\section{Micronization}

Micronization of drugs is done by milling techniques using Jet mill, Ball mill, Rotor-stator colloid mill, etc. This approach can reduce particle sizes down to 1 micron. Micronization improved the digestive absorption, and consequently the bioavailability and clinical efficacy of griseofulvin, progesterone, spironolactone and diosmin, ${ }^{9}$ Figure 1 presents the schematic presentation of Jet mill, which is commonly used for micronization.

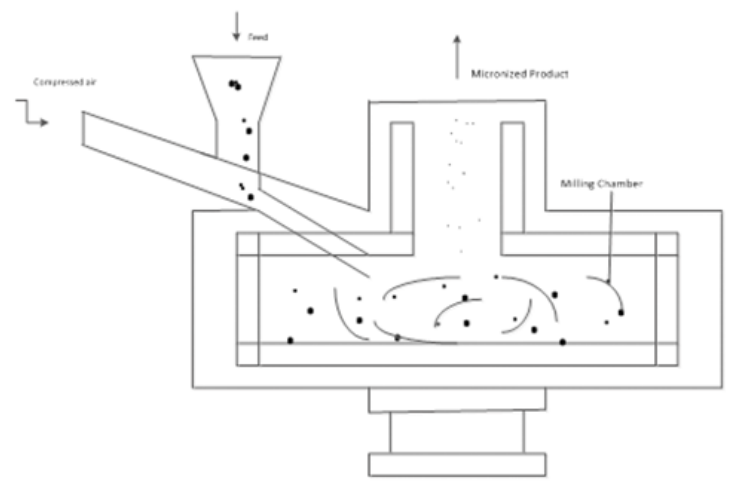

Figure I Schematic presentation of Jet Mill.

\section{Advantages}

a. Simple size reduction process

b. It increases the dissolution rate of drugs through increased surface area.

\section{Disadvantages}

a. It is not suitable for drugs having a high dose number because it does not change the saturation solubility of the drug.

b. It has limited applicability to range of compounds.

The following are some of the marketed products containing micronized drugs: Griseofulvin Tablets, Fenofibrate Capsules, Progesterone Capsules, Colestipol Tablets, Glyburide Tablets, and Estradiol tablets Nanosuspensions

Micron-sized particles do not sufficiently enhance the solubility of many poorly soluble drugs. In order to improve the dissolution or absorption characteristics further, drug particles can be reduced to the sub-micron or nano size ranges. Nanosuspension is a biphasic system consisting of nano sized drug particles (most typically around $100-200 \mathrm{~nm}$ ) stabilized by a surfactant. Nano-drugs enhance the bioavailability through the improvement of dissolution rate and saturation solubility of drugs, by virtue of their small sizes. They can be used for oral, topical or parenteral administration.

Nano size particles are generally prepared by "top down" 10 or "bottom up"11 methods. The top-down method involves breaking drug crystals into smaller ones by dry or wet milling. In the bottomup method, nanoparticles are produced by solution-based drug recrystallization. The bottom-up method requires careful maintenance of a supersaturated drug solution while inducing crystal nucleation, growth, and precipitation. When particles get smaller, and surface area becomes larger, cohesive and adhesive effects can lead to particle aggregation. This can be prevented by the addition of surfactants or polymers.

\section{Nanosuspensions can be prepared by the following methods}

a. Media/wet milling

b. High pressure homogenization

c. Precipitation technique

d. Combined precipitation and homogenization

Media milling and high pressure homogenization are the commonly used methods for the preparation of nanosuspensions. Nanosuspension can be converted in to dry powder by freeze drying or spray drying and then compressed in to a tablet or encapsulated in to a capsule. The liquid nanosuspension can also be sprayed on to sugar spheres to make drug layered pellets. Figure 2 shows the diagrammatic presentation of preparation of nanosuspension by high pressure homogenizer.

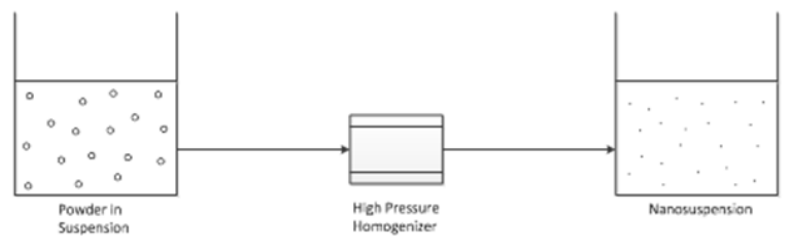

Figure 2 Diagramatic presentation of preparation of Nanosuspension by High pressure homogenizer.

\section{Advantages}

a. Rapid dissolution and improved bioavailability.

b. Can be given by multiple routes of administration (oral, parenteral, etc).

\section{Disadvantages}

a. Formulation design and stabilization during shelf life is more complex.

b. Lack of controlled release.

Table 1 presents the selected marketed products based on nanosuspensions

\section{Solid dispersions}

Solid dispersion consists of at least two different components, generally a stabilizing agent and a drug. Solid dispersion converts a crystalline drug in to an amorphous drug. In general, the amorphous version of a compound is more soluble in water, more hygroscopic and thermodynamically less stable compared to its crystalline counterpart. In a solid dispersion, the drug can be dispersed molecularly in the carrier system. Solid dispersions have shown promising bioavailability of poorly water-soluble drugs. ${ }^{4}$ The amorphous powders improve bioavailability by producing a high-energy form of the drug that functions by dissolving to form a supersaturated concentration in the intestine. One of the disadvantages of solid dispersion is related to its instability due to moisture and temperature. Several systems have shown changes in crystalline and a decrease in dissolution rate with aging. Spray drying and hot melt extrusion are two commonly employed methods to make solid dispersions of poorly soluble drugs.

\section{Spray drying}

Spray drying has been proven to improve the bioavailability of poorly soluble compounds. ${ }^{5}$ A spray dried dispersion (SDD) is an 
amorphous molecular dispersion of a drug in a polymer matrix created by dissolving drug and polymer in an organic solvent and then spraydrying the solution. For spray drying, the solubility of API in the solvent is crucial to ensure a readily scalable and viable process. The SDDs enhance the oral absorption of poorly-water soluble drugs by attaining and sustaining a supersaturated concentration of drug in the gastrointestinal fluid. SDDs are usually amenable for incorporation into a variety of final oral dosage forms, including capsules, tablets and sachets.

Frequently used polymers for SDDs are hydroxypropyl methyl cellulose acetate succinate (HPMC-AS), hydroxypropyl methyl cellulose (HPMC), polyvinylpyrrolidone (PVP), Copovidone, and methacrylate polymers (e.g., Eudragit L-100). HPMC-AS has unique properties that make it ideal for use in SDD including its high glass transition temperature $(\mathrm{Tg})$ in its unionized state, high solubility in volatile organic solvents, and its amphiphilic nature allowing insoluble molecules to interact with the hydrophobic regions of the polymer, whereas the hydrophilic regions allow these structures to remain as stable colloids in aqueous solution. ${ }^{5}$

In spray drying process, a poorly soluble drug and a suitable polymer is dissolved in a solvent such as acetone, ethyl alcohol or isopropyl alcohol. The drug-polymer solution is pumped to an atomizer inside the drying chamber of a spray drier. The solution is converted in to small droplets by the atomizer. The small droplets are rapidly converted to fine particles by the hot drying gas such as nitrogen. The dried powder is generally collected from the gas stream using a cyclone or filter system. Spray dried intermediate can be blended with a few other ingredients (e.g., diluent/glidant/lubricant) and can be compressed in to a tablet or encapsulated in to a capsule. Figure 3 illustrates the spray drying process.

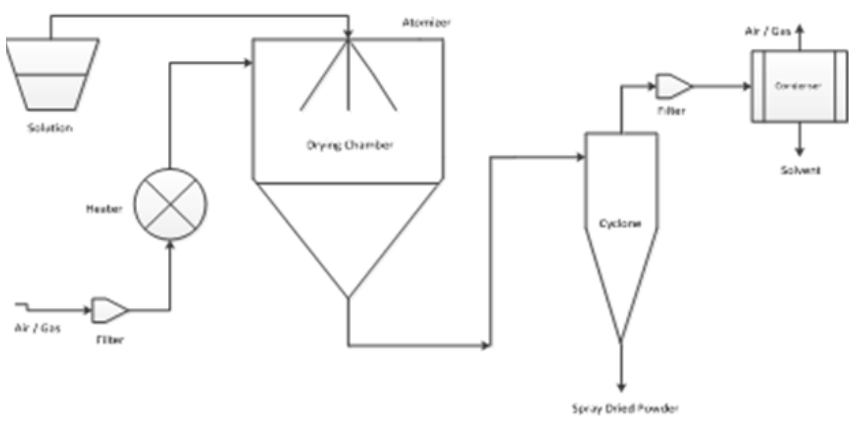

Figure 3 Spray drying process.

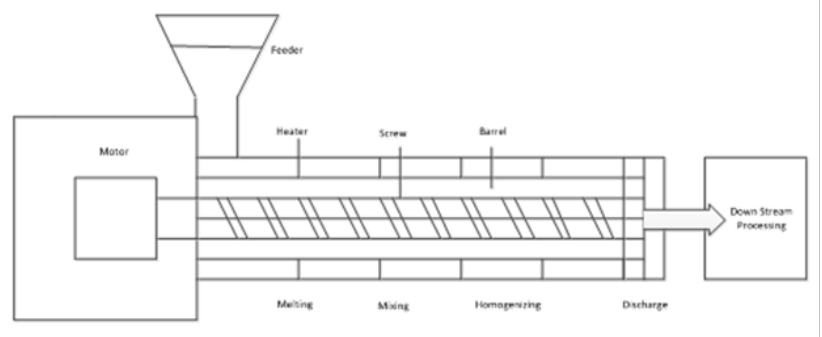

Figure 4 Schematic presentation of Hot Melt Extruder.

\section{Advantages}

a. A wide range of excipient and solvent choices makes it applicable to structurally diverse insoluble compounds with a wide range of physicochemical properties. b. The main advantage of spray drying over HME is the prevention of the thermal decomposition. In spray drying, a moderate temperature is used for a short period. So thermolabile drugs can be processed using spray drying.

c. Scalable process and standard oral solid dose manufacture.

\section{Disadvantages}

\section{a. Solvent based process.}

Table 2 Presents selected marketed products based on solid dispersions manufactured by spray drying

\section{Hot melt extrusion (HME)}

HME has been shown to molecularly disperse poorly soluble drugs in a polymer carrier, increasing dissolution rates and bioavailability. ${ }^{6}$ HME is a thermo-mechanical processing technique by which API is intimately or molecularly mixed with excipient carriers. The processing temperature is generally greater than melting or glass transition temperature to facilitate the formation of homogeneous dispersions or solutions of API in carrier system. An important requisite for the formation of solid dispersion by the HME method is the miscibility of the drug and the carrier in the molten form. Another important requisite is the thermal stability of both the drug and the carrier.

HME involves the application of heat, pressure and agitation through an extrusion channel to mix the drug and carrier together, and subsequently forcing them out through a die. HME enhances solubility by producing a stable, amorphous solid dispersion, increased-energy form through a combination of the process and the chemical properties of the excipient. The resulting product, called the extrudate, can be milled, blended with a few other ingredients (e.g., diluent, glidant, lubricant) and can be compressed in to a tablet or encapsulated in to a capsule. The commonly used polymers for HME are PVP, copovidone, HPMC, HPMC-AS, Methacrylate, and Polyethylene oxide.

Hot melt extruder consists of a feeding hopper, barrel, single or twin screw system, screw driving unit, and a heating or cooling device. Typical readouts include melt pressure, melt temperature, and motor amperage. Twin screw extruders utilize segmented screws that are assembled on high torque shafts. Barrels are also modular and integrate internal bores for cooling. Segmented screws/barrels, in combination with the controlled pumping and wiping characteristics of the co-rotating, self-wiping screws, allows screw/barrel geometries to be matched to the process tasks. Some of the process parameters to be controlled are screw speed, feed rate, temperature along the barrel and die, and vacuum level. Figure 4 presents the schematic diagram of hot melt extruder

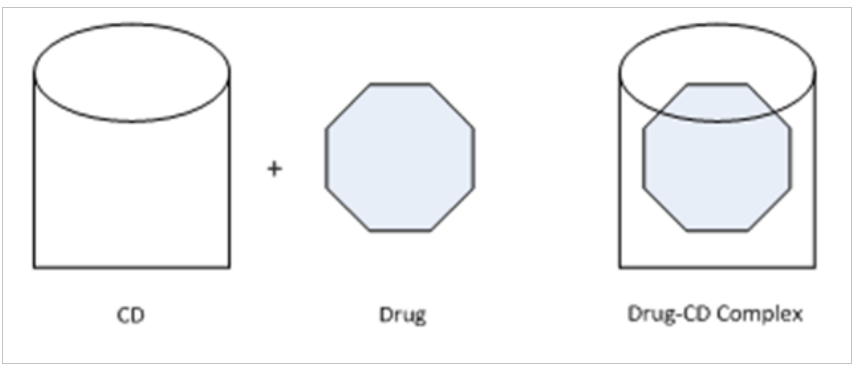

Figure 5 Drug-Cyclodextrin Inclusion Complex. 


\section{Advantages}

a. Solvent-free process.

b. Continuous processing allows for good process control and scalability.

c. Extrudate is versatile in its end use, including potential incorporation in controlled release delivery formulations.

\section{Disadvantages}

a. Limited applicability, requires thermally stable drugs and excipients.

Selected commercially available products based on solid dispersion manufactured by HME are presented in Table 3 .

Table I List of Selected Marketed Products Based on Nanosuspensions

\begin{tabular}{llll}
\hline Product /Drug & Process & Dosage form & Company \\
\hline Tricor (Fenofibrate) & Media/wet milling & Tablet & Abbott \\
Megace ES (Megesterol acetate) & Media milling & Liquid nanosuspension & Par Pharmaceutical \\
Rapamune (Sirolimus) & Media milling & Tablet & Wyeth \\
Emend (Aprepitant) & Media milling & Capsule & Merck \\
Triglide (Fenofibrate) & High pressure homogenization & Tablet & First Horizon Pharma \\
Invega Sustenna (Paliperidone Palmitate) & High pressure homogenization & Liquid nanosuspension & Johnson \& Johnson \\
\hline
\end{tabular}

Table 2 Selected marketed products based on solid dispersion (spray dried)

\begin{tabular}{lll}
\hline Product / Drug & Dispersion polymer/Carrier & Company \\
\hline Intelence (Etravirine) & HPMC & Janssen \\
Cesamet (Nabilone) & PVP & Meda \\
Incivek (Telaprevir) & HPMC-AS & Vertex \\
Kalydeco (Ivacaftor) & HPMC-AS & Vertex \\
Zortress (Everolimus) & HPMC & Novartis \\
\hline
\end{tabular}

Table 3 Selected commercially available products based on solid dispersion manufactured by HME

\begin{tabular}{lll}
\hline Product / Drug & Dispersion polymer/Carrier & Company \\
\hline Kaletra (Lopinavir \& Ritonavir) & PVP/PVA & Abbott \\
Isoptin SRE-240 (Verapamil) & Multiple & Soliqs \\
Rezulin (Troglitazone) & PVP & Pfizer \\
lbuprofen & Multiple & Soliqs \\
Noxafil (Posaconazole) & HPMC-AS & Merck \\
Onmel (Itraconazole) & HPMC & Sebala
\end{tabular}

\section{Lipid based delivery systems}

Lipid based formulations have been evaluated to enhance the solubility and bioavailability of poorly soluble drugs for a number of years. ${ }^{8}$ A critical early step is to establish the solubility range of the drug in various lipids. Once formed, the drug/lipid mixture can sustain drug concentrations in targeted environments. Lipid formulations produce complex structures comprising micelles, micro- or nanostructures, which are directly responsible for delivering the drug. Lipid formulations can be complex because of the number of additives required to meet performance objectives. The majority of the lipid based formulations are filled into soft or hard gelatin capsules. Lipid based formulations are generally liquids, but may also be solid or semi solid at room temperature when high melting lipids are used or when lipids are adsorbed on to a carrier.

The excipients employed to form lipid based formulations are generally from three categories - lipids, surfactants and cosolvents. The lipid formulation classification system classifies lipid based formulations into four main types (Type I to IV), based on the relative proportions of included lipids, surfactants and cosolvents. ${ }^{12}$ Many self-microemulsifying drug delivery system (SMEDDS) or self-nanoemulsifying drug delivery system (SNEDDS) formulations typically fall under type III formulations. Self-emulsifying drug delivery systems (SEDDS), SMEDDS and SNEDDS were explored in many studies as an efficient approach for improving the bioavailability and dissolution rate of poorly water-soluble drugs. One of the greatest advantages of incorporating poorly soluble drugs into such formulations is their spontaneous emulsification and formation of an emulsion, microemulsion or nanoemulsion in aqueous media. Lipid formulations provide an attractive option for enhancing the oral bioavailability of beyond the rule of 5 (b-r-o-5) compounds. Unlike many formulation approaches, they have the potential to address solubility, permeability and presystemic metabolic liabilities, although additional research is needed in this area.

\section{Advantages of lipid based formulations}

a. Can be used for a broad range of poorly soluble drugs.

b. provides advantage for drugs with inherently low melting points, where solid dose forms may be impractical.

c. Provides advantage for low dose compounds with potential content uniformity issues and for irritant

d. and toxic compounds where dust control is a challenge.

e. Excipients used in lipid based delivery systems may improve intestinal permeability, both via changes to passive permeability and via inhibition of efflux transporters.

f. Lipid based delivery systems significantly enhance the intestinal solubilisation of lipophilic poorly water soluble drugs. These increases exposure and in most cases also attenuates the large positive food effect commonly seen for poorly water soluble drugs after oral administration. 


\section{Disadvantages of lipid based formulations}

a. More complex to formulate due to a large number of variables that must be considered.

Table 4 lists the selected commercially available lipid based delivery systems.

\section{Inclusion complexes}

The drug and a companion molecule such as cyclodextrin form an inclusion complex. The drug resides in cavities of the companion molecule, bound by noncovalent intermolecular forces. The resultant formulation improves the solubility and bioavailability of poorly soluble drugs. ${ }^{7}$ The drug molecular size and lipophilicity are important factors to consider in the formulation of drug-cyclodextrin inclusion complexes. Drug-cyclodextrin inclusion complexes can be prepared by Coprecipitate method and Spray drying method. Figure 5 illustrates the formation of drug-cyclodextrin inclusion complex.

\section{Advantages}

a. Cyclodextrins enhance solubility and bioavailability of poorly soluble drugs.

b. Cyclodextrins facilitate aqueous stability.

\section{Disadvantages}

a. Drug loading and dosage form design may be limiting factors.

b. Scale up of manufacturing process can be a challenge.

A selected list of commercially available cyclodextrin based products is presented in Table 5 .

Table 4 List of selected commercially available lipid based delivery systems

\begin{tabular}{lll}
\hline Product/Drug & Lipid excipients & Dosage form \\
\hline Rocaltrol (Calcitriol) & Frac. triglyceride of coconut oil & Soft gelatin capsules \\
Absorica (Isotretinoin) & Multiple & Hard gelatin capsules \\
Neoral (Cyclosporine) & Cremophor RH 40 & Soft gelatin capsules \\
Depakene (Valproic acid) & Corn oil & Soft gelatin capsules \\
Fenogal (Fenofibrate) & Gelucire 44/14 & Hard gelatin capsules \\
Restandol (Testosterone) & Oleic acid & Soft gelatin capsules \\
Vesanoid (Tretinoin) & Beeswax, hydrogenated soybean oil & Soft gelatin capsules \\
Rapamune (Sirolimus) & Phosal 50 PG, & Oral solution \\
& Polysorbate 80 & \\
Fortovase (Saquinavir) & Medium-chain & \\
& mono- and diglycerides & Soft gelatin capsules \\
Prometrium (Progesterone) & Povidone & \\
\hline
\end{tabular}

Table 5 List of selected Marketed Cyclodextrin (CD) based products

\begin{tabular}{llll}
\hline Drug & Cyclodextrin & Dosage form & Company/Country \\
\hline Nimesulide & Beta-CD & Tablet & Novartis, Europe \\
Nicotine & Beta-CD & Sublingual tablet & Pharmacia, Sweden \\
Omeprazole & Beta-CD & Tablet & Betafarm, Germany \\
Aripiprazole & Sulfo butyl ether Beta-CD & i.m. solution & BMS, USA \\
Vericonazole & Sulfobutylether Beta-CD & i.v. solution & Pfizer, USA \\
Diclofenac Na & Hydroxypropyl Gamma-CD & Eye drop solution & Chauvin, France \\
Benexate & Beta-CD & Capsules & Teikoku/Japan \\
Piroxicam & Beta-CD & Tablet & Chiesi/ltaly \\
Nitroglycerin & Beta-CD & Sublingual tablet & Nippon \\
Cefotiam hexatil & & & Kayaku/Japan \\
hydrochloride & Alpha-CD & Tablet & Takeda/Japan \\
Itraconazole & 2-hydroxypropyl-Beta-CD & Oral solution and intramuscular injection & Janssen/Europe/USA \\
\hline
\end{tabular}

\section{Conclusion}

Majority of new chemical entities being developed currently are poorly soluble drugs. A number of poorly soluble drugs have been introduced to the market by using new technologies. Among the available technologies, solid dispersion formulations and lipid based formulations have shown the most commercial success and continue to be widely employed. Physicochemical characteristics of the drug and advantages and disadvantages of different technologies need to be considered before selecting a technology. Advances being made in solubility enhancement technologies and sophisticated analytical techniques to measure the performance of the dosage forms in-vitro and in-vivo and new research in the area of modeling and simulation are expected to facilitate the development of new dosage forms that will successfully overcome the limitations of not only poorly soluble drugs but also poorly permeable drugs.

\section{Acknowledgments}

None.

\section{Conflicts of interest}

This author has no conflict of interest in the content of this review article. 


\section{References}

1. Loftsson T, Brewster ME. Pharmaceutical applications of cyclodextrins: basic science and product development. $J$ Pharm Pharmacol. 2010;62(11):1607-1621.

2. Rasenack N, Muller BW. Micron-size drug particles:Common and novel micronization techniques. Pharm Dev Technol. 2004;9(1):1-13.

3. Gulsun T, Nelihan Gursoy R, Oner L. Nanocrystal technology for oral delivery of poorly water-soluble drugs. FABAD J Pharm Sci. 2009;34:5565 .

4. Duarte I, Temtem M, Gil M, et al. Overcoming poor bioavailability through amorphous solid dispersions. Industrial Pharmacy. 2011;30:4-6.

5. Friesen DT, Shanker R, Crew M, et al. Hydroxypropyl methylcellulose acetate-based spray-dried dispersions:An overview. Molecular Pharmaceutics. 2008;5(6):1003-1019.

6. Madan S, Madan S. Hot melt extrusion and its pharmaceutical applications. Asian J Pharm Sci. 2012;7(2):123-133.

7. Crestani de Miranda J, Martins TEA, Veiga F, et al. Cyclodextrins and ternary complexes:technology to improve solubility of poorly soluble drugs. Braz J Pharm Sci. 2011;47(4):665-681.

8. Feeney OM, Crum MF, McEvoy CL, et al. 50 years of oral lipid-based formulations:Provenance, progress and future perspective. Adv Drug Del Rev. 2016;101:167-194.

9. Chaumeil JC. Micronization:a method of improving the bioavailability of poorly soluble drugs. Methods Find Exp Clin Pharmacol. 1998;20(3):211215.

10. Suman K, Chandrasekar PVSR, Balajai S. Drug nanocrystals:A novel formulation approach for poorly soluble drugs. Int J Pharm Tech Res. 2009;1:682-694.

11. Liversidge EM, Liversidge GG, Cooper ER. Nanosizing:a formulation approach for poorly water soluble compounds. Eur J Pharm Sci. 2003;18(2):113-120.

12. Pouton CW. Formulation of poorly water-soluble drugs for oral administration: physicochemical and physiological issues and the lipid formulation classification system. Eur J Pharm Sci. 2006;29(3-4):278287. 